УДК 621.391

\author{
Оценка спектральной эффективности \\ и помехоустойчивости когерентного приема \\ незамирающего QBL-MSK-сигнала \\ В. Н. Поддубный ${ }^{1}$, В. В. Грибанов ${ }^{2}$, К. Ю. Ложкин ${ }^{3}$, \\ Д. Б. Соболев ${ }^{4}$, А. Н. Петренко ${ }^{5}$ Ю. И. Полтавец ${ }^{6}$

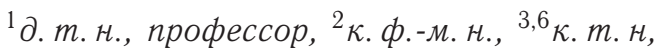 \\ ${ }^{1-3}$ ВУНЦ ВВС «Военно-воздушная академия им. Н. Е. Жуковского и Ю. А. Гагарина», Воронеж \\ ${ }^{4,5}$ Министерство обороны, Россия \\ ${ }^{6}$ АO «Российские космические системь» \\ e-mail:vgribanov@yandex.ru,k.yu.lozhkin@mail.ru
}

\begin{abstract}
Аннотация. В статье обосновывается математическая модель незамирающего QBL-MSK-сигнала, алгоритмы его формирования и приема. Найден энергетический спектр QBL-MSK-сигнала и разработан методический аппарат в виде аналитических методик и математических моделей оценки эффективности воздействия гауссовской помехи на когерентный приемник этого сигнала.

Отличительная особенность предлагаемых методик и моделей - учет межсимвольной интерференции, специально вводимой в сигнал на передающей стороне в процессе сглаживания фронтов передаваемых посылок. Показано, что сглаживание посредством QBL-импульса обеспечивает значительное сужение спектра сигнала и некоторое улучшение помехоустойчивости его приема.
\end{abstract}

Ключевые слова: QBL-MSK-сигнал, помехоустойчивость приема, энергетический спектр, спектральная и энергетическая эффективность, вероятность ошибочного приема, алгоритмы приема

\title{
Estimation of the Spectral Efficiency and Noise Immunity of the Coherent Reception of an Unfading QBL-MSK Signal
}

\author{
V. N. Poddubnyy ${ }^{1}$, V. V. Gribanov ${ }^{2}$, K. Yu. Lozhkin ${ }^{3}$, \\ D. B. Sobolev ${ }^{4}$, A. N. Petrenko ${ }^{5}$, Yu. I. Poltavets ${ }^{6}$ \\ ${ }^{1}$ doctor of engineering science, professor, ${ }^{2}$ candidate of physical and mathematical science, \\ ${ }^{3,6}$ candidate of engineering science, \\ ${ }^{1-3}$ Zhukovsky-Gagarin Air Force Academy, Voronezh \\ ${ }^{4,5}$ Ministry of Defence, Russia \\ ${ }^{6}$ Joint Stock Company "Russian Space Systems" \\ e-mail:vgribanov@yandex.ru,k.yu.lozhkin@mail.ru
}

\begin{abstract}
The mathematical model of an unfading QBL-MSK signal, as well as the algorithms for its formation and reception are substantiated in the article. The energy spectrum of the QBL-MSK signal is found, and a methodical apparatus is developed in the form of analytical techniques and mathematical models for estimating the effectiveness of the Gaussian noise interference on a coherent receiver of this signal.

A distinctive feature of the proposed methodologies and models is the consideration of intersymbol interference, which is specially introduced into the signal on the transmitting side in the process of leading edge smoothing of the transmitted packages. It is shown that the smoothing by a QBL-pulse provides a significant narrowing of the signal spectrum and some improvement in noise immunity of its reception.
\end{abstract}

Keywords: QBL-MSK signal, noise immunity, energy spectrum, spectral and energy efficiency, reception error probability, reception algorithms 
Спектрально и энергетически эффективные частотно-манипулированные сигналы с минимальным сдвигом (ЧМ-MC-сигналы, MSK-сигналы В иностранной литературе) находят широкое применение в современных системах передачи различных потоков информации в цифровой форме. Несмотря на большое число научно-технических публикаций, посвященных исследованию различных аспектов построения, функционирования и оценки эффективности систем передачи информации ЧМ-MC-сигналами, в настоящее время недостаточно внимания уделяется теоретическим оценкам спектральной эффективности и помехоустойчивости таких сигналов с частичным откликом, в частности QBL-MSK-сигналам (Quasibandlimited minimum shift keying - ЧМ-МС-сигнал с квазиограниченной полосой).

Цель работы - получить аналитические выражения для энергетического спектра и оценки помехоустойчивости QBL-MSK-сигнала и выполнить анализ этих выражений.

\section{Математическая модель QBL-MSK-сигнала}

Пусть в системе передачи информации используется квадратурный способ формирования рассматриваемого ЧМ-MC-сигнала. В этом случае для передачи $n$ двоичных элементов (битов) $c_{i}=$ $=0,1, i=\overline{1, n}$ используется $[1,2] n+1$ двоичных элементов $d_{i}=0,1, i=\overline{0, n}$, включающих один опорный $d_{0}=0,1$ и $n$ перекодированных по модулю 2 элементов $d_{i}=d_{i-1} \oplus c_{i} i \geqslant 1$, из которых формируются биполярные импульсы (посылки) длительностью $T_{c}$

$$
a_{i}=-(-1)^{d_{i}}
$$

Последовательность биполярных импульсов $a_{i}$ (1) подается на вход демультиплексора (рис. 1), который разбивает ее на 4 подпоследовательности $a_{4 k+l}(l=0,1,2,3 ; k=0,1,2, \ldots)$, где в каждой из четырех подпоследовательностей содержится каждый четвертый бит последовательности (1). Далее расширители импульсов увеличивают длительность импульсов в каждом канале с $T_{c}$ до $4 T_{c}$ и в каждом канале происходит умно- жение этих удлиненных прямоугольных импульсов $b_{4 k+l}$ на сглаживающую функцию QBL-импульca $g_{\mathrm{QBL}}\left(t-(4 k+l) T_{c}\right)$ :

$g_{\mathrm{QBL}}(t)=\left\{\begin{array}{l}\left(\frac{\sin \left(\pi\left(t-2 T_{c}\right) /\left(2 T_{c}\right)\right)}{\pi\left(t-2 T_{c}\right) /\left(2 T_{c}\right)}\right)^{3}, 0<t<4 T_{c} . \\ 0, \quad t<0 \text { или } t>4 T_{c} .\end{array}\right.$

Указанная QBL-функция формируется фильтром с конечной импульсной характеристикой (КИХ-фильтром), на вход которого поступают периодические $\delta$-импульсы вида $\delta\left(t-4 k T_{c}\right)$. На выходе KИХ-фильтра генерируются QBL-импульсы (функции) $g_{\mathrm{QBL}}\left(t-4 k T_{c}\right)$, которые перед умножением на удлиненные до $4 T_{c}$ элементы последовательности $b_{4 k+l}$ задерживаются на время $l T_{c}, l=$ $=0,1,2,3$. Таким образом, в каждом из 4 каналов происходит формирование биполярных QBL-импульсов $g\left(t-(4 k+l) T_{c}\right)$ вида

$$
g\left(t-(4 k+l) T_{c}\right)=b_{4 k+l} g_{\mathrm{QBL}}\left(t-(4 k+l) T_{c}\right) .
$$

Далее QBL-импульсы четырех каналов попарно суммируются, образуя два канала - четный (синусный или квадратурный $(Q)) u_{Q}(t)$ и нечетный (косинусный или синфазный $(I)) u_{I}(t)$, где четный канал содержит сумму импульсов $g(t-$ $\left.-(4 k+l) T_{c}\right)$ с $l=0$ и $l=2$, а нечетный содержит сумму импульсов $g\left(t-(4 k+l) T_{c}\right)$ с $l=1$ и $l=3$. Затем QBL-импульсы в четном и нечетном каналах умножаются на гармонические несущие с частотой $\omega_{0}$ и начальной фазой $\varphi_{0}$ так, что сигналы в четном (синусном) и нечетном (косинусном) каналах имеют вид

$$
\begin{gathered}
s_{Q}(t)=u_{Q}(t) \sin \left(\omega_{0} t+\varphi_{0}\right), \\
s_{I}(t)=u_{I}(t) \cos \left(\omega_{0} t+\varphi_{0}\right), \\
u_{Q}(t)=\sum_{k} g\left(t-2 k T_{c}\right)=\sum_{k} b_{2 k} g_{\mathrm{QBL}}\left(t-2 k T_{c}\right), \\
u_{I}(t)=\sum_{k} g\left(t-(2 k+1) T_{c}\right)= \\
=\sum_{k} b_{2 k+1} g_{\mathrm{QBL}}\left(t-(2 k+1) T_{c}\right),
\end{gathered}
$$

где индекс суммирования принимает значения $k=$ $=0,1,2, \ldots$ 


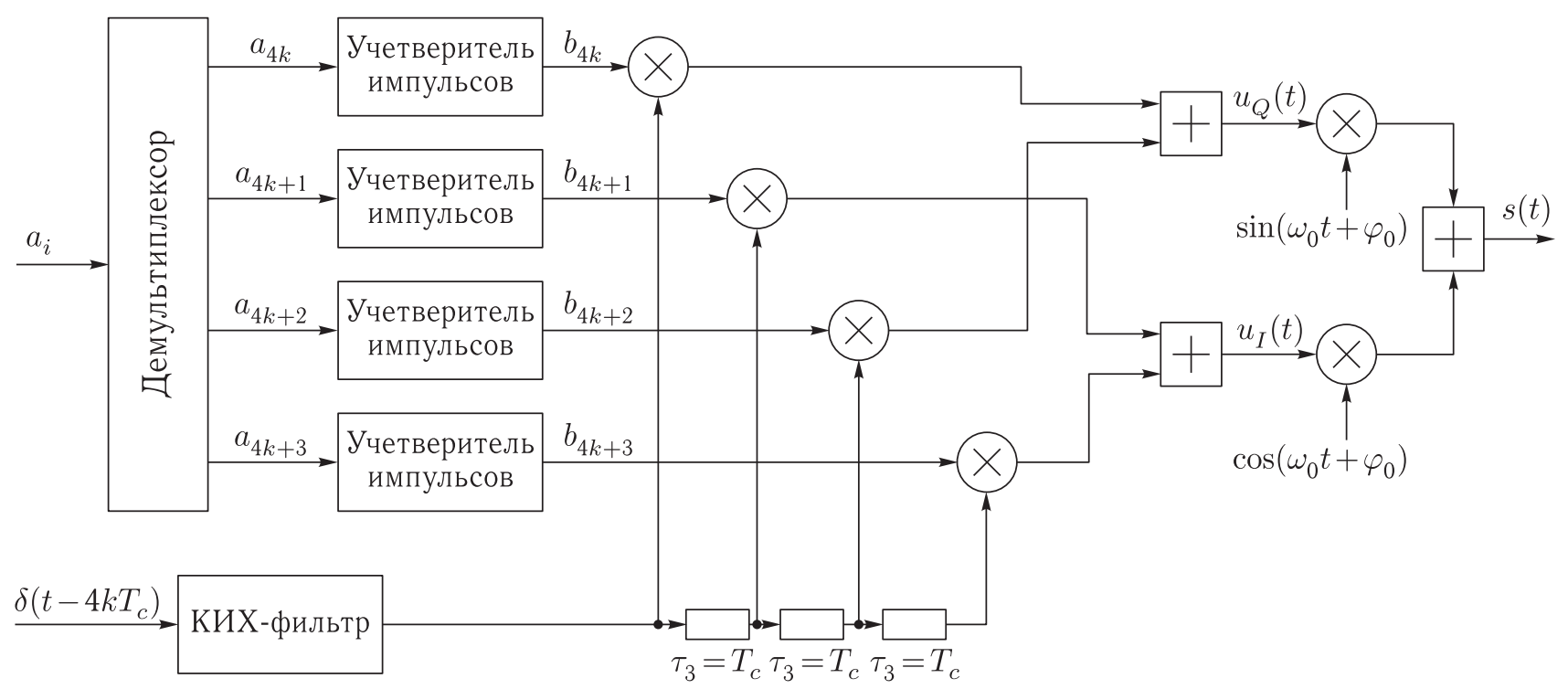

Рис. 1. Схема устройства формирования QBL-MSK-сигнала

На выходе формирователя QBL-MSK-сигнала (модулятора) сигналы синусного и косинусного каналов складываются и результирующий выходной сигнал на каждом тактовом интервале длительностью $T_{c}$ (с учетом усиления усилителем мощности и антенной) определяется выражением $s(t)=U_{0}\left[u_{I}(t) \cos \left(\omega_{0} t+\varphi_{0}\right)+u_{Q}(t) \sin \left(\omega_{0} t+\varphi_{0}\right)\right]$,

где $U_{0}-$ амплитуда сигнала.

\section{Помехоустойчивость приема незамирающего QBL-MSK-сигнала}

Пусть на входе приемника действует аддитивная смесь $x(t)$ незамирающего QBL-MSK-сигнала $s(t)(3)$ и помехи в виде белого шума $n(t)$ с односторонним энергетическим спектром $N_{0}$ и математическим ожиданием $\langle n(t)\rangle=0$ :

$$
x(t)=s(t)+n(t)=s_{c}(t)-s_{s}(t)+n(t) .
$$

Вследствие общности математических моделей формирования QBL-MSK и гармонического ЧМ-MC-сигналов [3], для приема незамирающего QBL-MSK-сигнала используем приемник (см. рис. 2), синтезированный на основании [4] для приема гармонического ЧМ-МС-сигнала. В этом приемнике реализуется следующий алгоритм приема $i$-го двоичного элемента $c_{i}$

$$
b_{i} b_{i-1} \stackrel{c_{i}=1}{<} \underset{c_{i}=0}{\gtrless} f_{\text {пор }}=0,
$$

причем напряжения $b_{i}$ определяются выражением

$$
b_{i}=\int_{(i+1) T_{c}}^{(i+3) T_{c}} x(t) u_{\mathrm{O \Pi}_{i}}(t) d t,
$$

$$
\text { где } u_{\mathrm{O}_{i}}(t)=\left\{\begin{array}{l}
u_{\mathrm{O}_{S}}(t) \text { для четных } i \\
u_{\mathrm{O} \Pi_{C}}(t) \text { для нечетных } i
\end{array}\right.
$$

и $i=2 k$ для четных $i$ и $i=2 k+1$ для нечетных $i$, $k=0,1,2, \ldots$.

Найдем числовые характеристики напряжений $b_{i}(6)$, необходимые для оценки помехоустойчивости рассматриваемого приемника. Из (4) и (6) следует, что напряжения $b_{i}$ для любого значения $i$ являются нормальными случайными независимыми величинами. Для нахождения математических ожиданий и дисперсий этих случайных величин применим подход, используемый в [3] для нахождения числовых характеристик определенных интегралов от случайных процессов. При этом необходимо принять во внимание тот факт, что QBL-MSK-сигнал является сигналом с неполным 


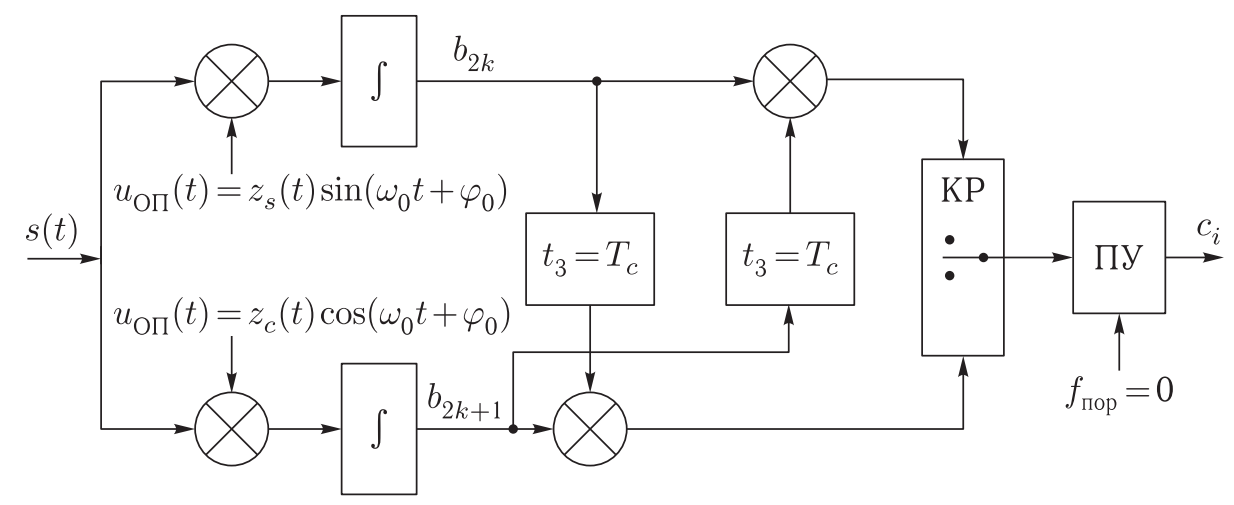

Рис. 2. Структурная схема когерентного приемника QBL-MSK-сигнала
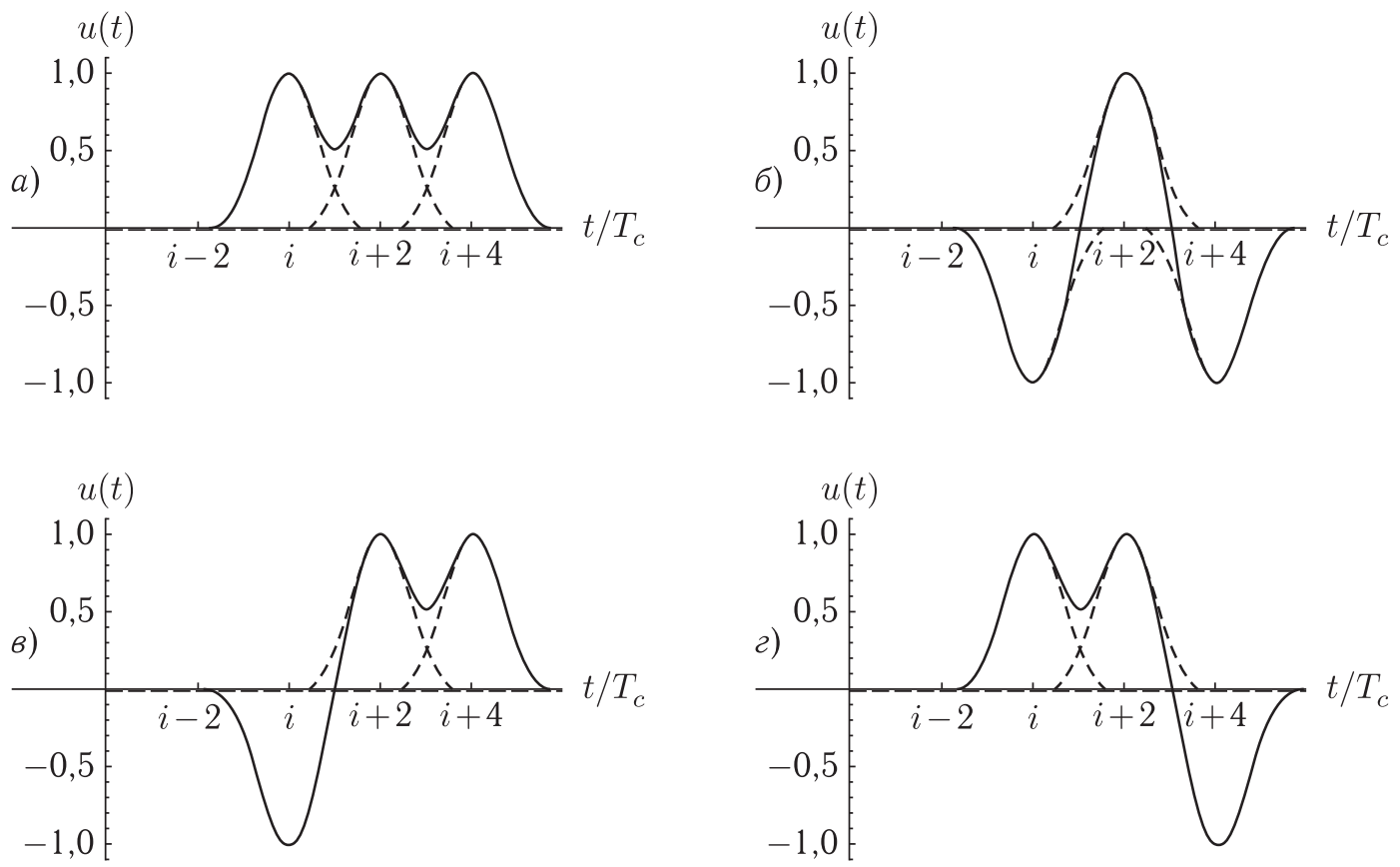

Рис. 3. Варианты поведения сглаживающих функций двоичных биполярных импульсов на трех соседних тактовых интервалах

откликом, сглаживающая функция биполярного импульса которого на каждом тактовом интервале содержит вклады от трех независимых функций: сглаживающей функции импульса на данном $i$-м тактовом интервале и сглаживающих функций двух импульсов на соседних тактовых интервалах. На рис. 3 показаны 4 возможных ситуации в случае положительного импульса $b_{i}$ : знаки биполярных импульсов на всех трех интервалах совпадают (рис. 3,a); знаки боковых биполярных импульсов противоположны знаку центрального импульса (рис. 3,б); знак центрального биполярного импульса совпадает со знаком только одного бокового импульса (рис. 3, в и 3, г).

На рис. 3 средний сглаживающий импульс имеет порядковый номер $i$, независимо от того, четно $i(i=2 k)$ или нечетно $(i=2 k+1)$, где $k=$ $=0,1,2, \ldots$ Сглаживающий $i$-й по счету импульс начинается с тактового момента $i$, а заканчивается на тактовом моменте $i+4$. Максимум $i$-го импульса достигается на тактовом моменте $i+2$, что соответствует изображенным на рис. 3 вариантам поведения сглаживающих функций. На основании изложенного нетрудно изобразить по аналогии 
четыре варианта поведения трех соседних сглаживающих функций, если средняя функция будет отрицательна.

Вначале найдем средние значения (математические ожидания) случайных величин $b_{i}(6)$

$\left\langle b_{i}\right\rangle=\left\langle\int_{(i+1) T_{c}}^{(i+3) T_{c}} x(t) u_{\mathrm{O}_{i}}(t) d t\right\rangle=\int_{(i+1) T_{c}}^{(i+3) T_{c}} s(t) u_{\mathrm{O}_{i}}(t) d t$.

Учитывая, что $\langle n(t)\rangle=0$, а несущие $\sin \left(\omega_{0} t+\right.$ $\left.+\varphi_{0}\right)$ и $\cos \left(\omega_{0} t+\varphi_{0}\right)$ ортогональны на временно́м интервале длительностью $2 T_{c}$, для четырех рассматриваемых ситуаций имеем

$$
\begin{gathered}
\left\langle b_{11, i}\right\rangle=\beta_{11} b_{i} U_{0} T_{c} / 2, \\
\left\langle b_{00, i}\right\rangle=\beta_{00} b_{i} U_{0} T_{c} / 2, \\
\left\langle b_{01, i}\right\rangle=\left\langle b_{10, i}\right\rangle=\beta_{01} b_{i} U_{0} T_{c} / 2,
\end{gathered}
$$

где нижними индексами $11,01,10$ и 00 обозначены ситуации, соответствующие рис. $3, a, 6,6$ и 3, г соответственно, а значения параметров $\beta$ равны:

$$
\begin{aligned}
& \beta_{11}= \\
& =\int_{1}^{3}\left(\widetilde{g}_{\mathrm{QBL}}(t+2)+\widetilde{g}_{\mathrm{QBL}}(t)+\widetilde{g}_{\mathrm{QBL}}(t-2)\right)^{2} d t=1,21 ; \\
& \beta_{10}=\int_{1}^{3}\left(\left(\widetilde{g}_{\mathrm{QBL}}(t+2)+\widetilde{g}_{\mathrm{QBL}}(t)-\widetilde{g}_{\mathrm{QBL}}(t-2)\right) \times\right. \\
& \left.\times\left(\widetilde{g}_{\mathrm{QBL}}(t+2)+\widetilde{g}_{\mathrm{QBL}}(t)+\widetilde{g}_{\mathrm{QBL}}(t-2)\right)\right) d t=1,13 ; \\
& \beta_{01}=\int_{1}^{3}\left(\left(-\widetilde{g}_{\mathrm{QBL}}(t+2)+\widetilde{g}_{\mathrm{QBL}}(t)+\widetilde{g}_{\mathrm{QBL}}(t-2)\right) \times\right. \\
& \left.\times\left(\widetilde{g}_{\mathrm{QBL}}(t+2)+\widetilde{g}_{\mathrm{QBL}}\left(t T_{c}\right)+\widetilde{g}_{\mathrm{QBL}}(t-2)\right)\right) d t=1,13 ; \\
& \beta_{00}=\int_{1}^{3}\left(\left(-\widetilde{g}_{\mathrm{QBL}}(t+2)+\widetilde{g}_{\mathrm{QBL}}(t)-\widetilde{g}_{\mathrm{QBL}}(t-2)\right) \times\right. \\
& \left.\times\left(\widetilde{g}_{\mathrm{QBL}}(t+2)+\widetilde{g}_{\mathrm{QBL}}(t)+\widetilde{g}_{\mathrm{QBL}}(t-2)\right)\right) d t=1,06,
\end{aligned}
$$

где

$$
\begin{aligned}
\tilde{g}_{\mathrm{QBL}}(t) & =g_{\mathrm{QBL}}\left(t T_{c}\right)= \\
& =\left\{\begin{array}{l}
\left(\frac{\sin (\pi(t-2) / 2)}{\pi(t-2) / 2}\right)^{3}, \quad 0<t<4 ; \\
0, \quad t<0 \text { или } t>4 .
\end{array}\right.
\end{aligned}
$$

Дисперсии случайных величин определяются выражением

$$
\sigma_{i}^{2}=\left\langle\left[\int_{(i+1) T_{c}}^{(i+3) T_{c}} n(t) u_{\mathrm{O \Pi}_{i}}(t) d t\right]^{2}\right\rangle .
$$

Записывая квадрат любого из интегралов (10) как двукратный интеграл с переменными интегрирования $t_{1}$ и $t_{2}$, меняя местами операции усреднения и интегрирования, учитывая, что $\left\langle n\left(t_{1}\right) n\left(t_{2}\right)\right\rangle=\left(N_{0} / 2\right) \delta\left(t_{2}-t_{1}\right)$, а также применяя фильтрующее свойство $\delta$-функции $\delta\left(t_{2}-t_{1}\right)$, окончательно получим

$$
\sigma_{11, i}^{2}=\sigma_{00, i}^{2}=\sigma_{01, i}^{2}=\sigma_{10, i}^{2}=\sigma^{2}=\beta_{11} N_{0} T_{c} / 4 .
$$

При нахождении вероятностей $\bar{p}_{0}$ и $\bar{p}_{1}$ искажения двоичных элементов $c_{i} \in\{0,1\}$ воспользуемся полученными числовыми характеристиками случайных величин $b_{i}$ и обозначим вероятности того, что знаки случайных величин $b_{i}$ будут соответствовать знакам их математических ожиданий, через $\dot{P}_{i}$, а вероятности несовпадения этих знаков - через $\bar{P}_{i}=1-\dot{P}_{i}$. Тогда вероятность искажения элемента $b_{i}$ равна

$$
\bar{P}_{i}=\frac{1}{4} \sum_{k \in x} \bar{P}_{k, i},
$$

где $x=\{11,10,01,00\}$, а $\bar{P}_{k, i}$ обозначает вероятность совпадения знаков случайных величин $b_{i}$ с их математическими ожиданиями в каждой из 4 возможных ситуаций.

Из (5), (7) и (11) следует, что вероятности $\bar{p}_{0}$ и $\bar{p}_{1}$ одинаковы: $\bar{p}_{0}=\bar{p}_{1}=\bar{p}$. Эти вероятности на основании (5) определяются формулой

$$
\begin{aligned}
\bar{p}=\bar{P}_{i} \dot{P}_{i-1}+\dot{P}_{i} \bar{P}_{i-1}= & 2 \bar{P}_{i}\left(1-\bar{P}_{i}\right)= \\
& =\frac{1}{8} \sum_{k, n \in x} \bar{P}_{k, i}\left(1-\bar{P}_{n, i}\right),
\end{aligned}
$$

где $x=\{11,10,01,00\}$.

Так как вероятности $\bar{P}_{k, i}$ не зависят от номера интервала, что следует из (7) и (11), то $\bar{P}_{k, i}=\bar{P}_{k}$, и окончательно получаем

$$
\bar{p}=\frac{1}{8} \sum_{k, n \in x} \bar{P}_{k}\left(1-\bar{P}_{n}\right)
$$


причем вероятность $\bar{P}_{k}$ определяется на основании (8) и (11) выражением

$$
\bar{P}_{k}=\frac{1}{2}\left(1-\operatorname{erf}\left(\frac{\beta_{k}}{\sqrt{\beta_{11} h}}\right)\right),
$$

в котором $\operatorname{erf}(y)=(2 / \sqrt{\pi}) \int_{0}^{y} e^{-t^{2}} d t, h=2 \sigma_{\Pi}^{2} / U_{0}^{2}$, где $\sigma_{\Pi}^{2}=N_{0} / T_{c}-$ мощность гауссовской помехи $n(t)$ в полосе $\Delta f=1 / T_{c}$.

Из сравнения (12) и (13) с аналогичными выражениями для приемников классического ЧММС-сигнала [5], ЧМ-МС-сигнала, сформированного на основе частотной манипуляции с непрерывной фазой [6], а также гармонического ЧМMC-сигнала [3] и сигнала с четырехпозиционной фазовой манипуляцией (ФМ-4-сигнала) [9] следует, что по энергетической эффективности (по помехоустойчивости) QBL-MSK-сигнал практически не отличается от перечисленных выше сигналов, если мощности и скорости передачи информации этих сигналов будут одними и теми же, а спектральная плотность мощности гауссовской помехи будет равна $N_{0}$. Так, для достижения значения вероятности искажения двоичного элемента $\bar{p}=0,2$ (соответствующей полному разрушению информационного содержания передаваемого сообщения [10]) для QBL-MSK сигнала необходимо обеспечить соотношение помеха/сигнал $h=1,44$, в то время как для остальных сигналов это соотношение равно $h=1,36$.

\section{Энергетический спектр QBL-MSK-сигнала}

Спектральная функция (спектр) сигнала (3), взятого на интервале времени $(N+5) T$, где $N-$ четное число, определяется выражением [7]

$$
\begin{aligned}
& \dot{S}(\omega)= \\
& =\int_{0}^{(N+5) T} U_{0}\left[u_{I}(t) \cos \left(\omega_{0} t\right)-u_{Q}(t) \sin \left(\omega_{0} t\right)\right] \mathrm{e}^{-\mathrm{j} \omega t} d t= \\
& =\int_{0}^{(N+5) T} \sum_{k} b_{2 k+1} g_{\mathrm{QBL}}\left(t-(2 k+1) T_{c}\right) \times \\
& \quad \times \cos \left(\omega_{0} t\right) \mathrm{e}^{-\mathrm{j} \omega t} d t+
\end{aligned}
$$

$$
\begin{aligned}
+\int_{0}^{(N+5) T} \sum_{k} b_{2 k} g_{\mathrm{QBL}}\left(t-2 k T_{c}\right) \sin \left(\omega_{0} t\right) \mathrm{e}^{-\mathrm{j} \omega t} d t= \\
=\dot{S}_{c}(\omega)+\dot{S}_{s}(\omega),
\end{aligned}
$$

где $k=0,1,2, \ldots, g_{\mathrm{QBL}}(t)$ определяется выражением (2), а начальная фаза сигнала без нарушения общности анализа выбрана нулевой: $\varphi_{0}=0$.

Для нахождения спектра $\dot{S}(\omega)$ воспользуемся подходом, изложенным в $[3,6,8]$. Для первого интеграла в (15) имеем

$$
\begin{aligned}
& \dot{S}_{c}(\omega)=U_{0} \sum_{k=0}^{N / 2} b_{2 k+1} \times \\
& \quad \times \int_{(2 k+1) T_{c}}^{(2 k+5) T_{c}} g_{\mathrm{QBL}}\left(t-(2 k+1) T_{c}\right) \cos \left(\omega_{0} t\right) \mathrm{e}^{-\mathrm{j} \omega t} d t .
\end{aligned}
$$

Заменим функцию $\cos \left(\omega_{0} t\right)$ формулой Эйлера $\cos \left(\omega_{0} t\right)=\left(e^{\mathrm{j} \omega_{0} t}+\mathrm{e}^{-\mathrm{j} \omega_{0} t}\right) / 2$, введем новую переменную интегрирования $x=t / T_{c}-2 k-3$ и обозначим $\Delta \omega=\omega-\omega_{0}$. В этом случае спектр $\dot{S}_{c}(\omega)$ для $\omega \geqslant 0$ принимает вид

$$
\dot{S}_{c}(\omega \geqslant 0)=\frac{U_{0} T_{c}}{2} F\left(\Delta \omega T_{c}\right) \sum_{k=0}^{N / 2} b_{2 k+1} \mathrm{e}^{-\mathrm{j} \Delta \omega(2 k+3) T_{c}},
$$

где

$$
F\left(\Delta \omega T_{c}\right)=\int_{-2}^{2} \widetilde{g}_{\mathrm{QBL}}(t+2) \mathrm{e}^{-\mathrm{j} \Delta \omega T_{c} t} d t,
$$

а функция $\widetilde{g}_{\mathrm{QBL}}(t)$ определена в (9).

При нахождении спектра $\dot{S}_{s}(\omega)$ воспользуемся формулой Эйлера $\sin \left(\omega_{0} t\right)=-\mathrm{j}\left(e^{\mathrm{j} \omega_{0} t}-\mathrm{e}^{-\mathrm{j} \omega_{0} t}\right) / 2$ и введем новую переменную интегрирования $x=$ $=t / T_{c}-2 k-2$. Тогда спектр $\dot{S}_{s}(\omega)$ для положительных частот принимает вид

$$
\begin{aligned}
& \dot{S}_{s}(\omega \geqslant 0)= \\
& =U_{0} \sum_{k=0}^{N / 2} b_{2 k} \int_{2 k T}^{(2 k+4) T} g_{\mathrm{QBL}}\left(t-2 k T_{c}\right) \sin \left(\omega_{0} t\right) \mathrm{e}^{-\mathrm{j} \omega t} d t= \\
& =\frac{\mathrm{j} U_{0} T_{c}}{2} F\left(\Delta \omega T_{c}\right) \sum_{k=0}^{N / 2} b_{2 k} \mathrm{e}^{-\mathrm{j} \Delta \omega(2 k+2) T_{c}},
\end{aligned}
$$


Подставляя (16) и (18) в (15), получим выражение спектра ЧМ-MC-сигнала (3) для положительных частот $(\omega \geqslant 0)$ в виде

$$
\begin{aligned}
\dot{S}(\omega & \geqslant 0)=\frac{U_{0} T_{c}}{2} F\left(\Delta \omega T_{c}\right) \times \\
& \times \sum_{k=0}^{N / 2} \mathrm{e}^{-\mathrm{j} \Delta \omega(2 k+1) T_{c}\left(\mathrm{j} b_{2 k}+b_{2 k+1} \mathrm{e}^{-\mathrm{j} \Delta \omega T_{c}}\right) .}
\end{aligned}
$$

На основании (19) и равенства Парсеваля [7] запишем выражение односторонней спектральной плотности мощности (физического энергетического спектра) QBL-MSK-сигнала (3) в виде

$$
G_{\phi}(\omega)=\lim _{N \rightarrow \infty} \frac{2}{(N+2) T_{c}}\left\langle|\dot{S}(\omega \geqslant 0)|^{2}\right\rangle,
$$

где усреднение ведется по времени всех $N+2$ тактовых импульсов, присутствующих в сумме (19), $\lim$ - символ нахождения предела, $\langle\cdot\rangle-$ символ усреднения по случайным значениям посылок $b_{i}$ и $|\cdot|-$ символ взятия модуля.

Воспользовавшись (19), получим

$$
\begin{aligned}
& \left\langle|\dot{S}(\omega \geqslant 0)|^{2}\right\rangle=\langle\dot{S}(\omega \geqslant 0) \stackrel{*}{S}(\omega \geqslant 0)\rangle= \\
& =U_{0}^{2} T_{c}^{2}\left|F\left(\Delta \omega T_{c}\right)\right|^{2} / 4 \times
\end{aligned}
$$

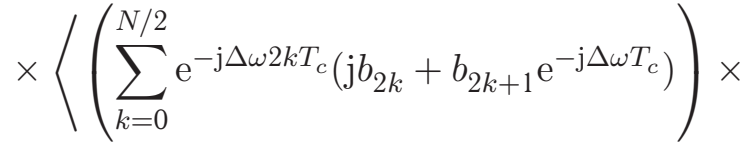

$$
\begin{aligned}
& \left.\times\left(\sum_{p=0}^{N / 2} e^{\mathrm{j} \Delta \omega 2 p T_{c}}\left(-\mathrm{j} b_{2 p}+b_{2 p+1} e^{\mathrm{j} \Delta \omega T_{c}}\right)\right)\right\rangle,
\end{aligned}
$$

где $\stackrel{*}{S}(\omega \geqslant 0)-$ спектр, сопряженный со спектром $\dot{S}(\omega \geqslant 0)$.

Если заменить в (21) среднее от суммы суммой средних и учесть, что для равновероятных и попарно независимых случайных величин $b_{2 k}$, $b_{2 k+1}, k=0,1, \ldots, N / 2$ выполняются соотношения

$$
\left\langle b_{2 k} b_{2 p}\right\rangle=\left\langle b_{2 k+1} b_{2 p+1}\right\rangle=\delta_{k, p}, \quad\left\langle b_{2 k} b_{2 p+1}\right\rangle=0,
$$

где $\delta_{p, k}$ - символ Кронекера, то в этом случае выражение (21) принимает вид

$$
\left\langle|\dot{S}(\omega \geqslant 0)|^{2}>=\frac{U_{0}^{2} T_{c}^{2}}{4}\left|F\left(\Delta \omega T_{c}\right)\right|^{2} 2 \cdot \frac{N+2}{2} .\right.
$$

Подставляя (22) в (20) и вычисляя предел при $N \rightarrow \infty$, получим

$$
G_{\phi}(\omega)=\frac{U_{0}^{2} T_{c}}{2}\left|F\left(\Delta \omega T_{A}\right)\right|^{2} .
$$

Спектр $F\left(\Delta \omega T_{c}\right)$ в (23) определяется интегралом (17), вычисление которого дает

$$
\begin{aligned}
F\left(\Delta \omega T_{c}\right) & =\int_{-2}^{2}\left(\frac{\sin (\pi t / 2)}{\pi t / 2}\right)^{3} \cos \left(\Delta \omega T_{c} t\right) d t= \\
& =\frac{1}{4 \pi^{3}}\left(\left(2 \Delta \omega T_{c}+3 \pi\right)^{2} \operatorname{Si}\left(2 \Delta \omega T_{c}+3 \pi\right)-\right. \\
& -3\left(2 \Delta \omega T_{c}+\pi\right)^{2} \operatorname{Si}\left(2 \Delta \omega T_{c}+\pi\right)+ \\
& +3\left(2 \Delta \omega T_{c}-\pi\right)^{2} \operatorname{Si}\left(2 \Delta \omega T_{c}-\pi\right)- \\
& \left.-\left(2 \Delta \omega T_{c}-3 \pi\right)^{2} \operatorname{Si}\left(2 \Delta \omega T_{c}-3 \pi\right)\right),
\end{aligned}
$$

где $\operatorname{Si}(z)$ - интегральная функция синуса, $\operatorname{Si}(z)=$ $=\int_{0}^{z} \frac{\sin (t)}{t} d t$

После подстановки $\Delta \omega=2 \pi\left(f-f_{0}\right)=2 \pi \Delta f$ и (24) в (23) получим окончательное выражение для энергетического спектра рассматриваемого QBL-MSK-сигнала

$$
\begin{aligned}
G_{\phi}(f) & =\frac{\ni}{16 \pi^{2}}\left\{\left(4 \Delta f T_{c}+3\right)^{2} \operatorname{Si}\left[\pi\left(4 \Delta f T_{c}+3\right)\right]-\right. \\
& -3\left(4 \Delta f T_{c}+1\right)^{2} \operatorname{Si}\left[\pi\left(4 \Delta f T_{c}+1\right)\right]+ \\
& \left.+3\left(4 \Delta f T_{c}-1\right)^{2} \operatorname{Si}\left[\pi\left(4 \Delta f T_{c}-1\right)\right]\right)- \\
& \left.-\left(4 \Delta f T_{c}-3\right)^{2} \operatorname{Si}\left[\pi\left(4 \Delta f T_{c}-3\right)\right]\right\}^{2}
\end{aligned}
$$

где $\ni=U_{0}^{2} T_{c} / 2-$ энергия посылки сигнала длительностью $T_{c}$.

На рис. 4 сплошной линией изображена зависимость физического энергетического спектра (25) QBL-MSK-сигнала от $\Delta f T_{c}$. На рисунке изображены заимствованные из $[3,6,8,9]$ зависимости физических энергетических спектров от $\Delta f T_{c}$ для гармонического ЧМ-MC-сигнала (штрихпунктирная линия), классического ЧМ-MC-сигнала и ЧМ-МС-сигнала, сформированного на основе частотной манипуляции с непрерывной фазой (точечная линия), а также для четырехпозиционного фазоманипулированного (ФМ-4) сигнала (пунктирная линия). Ширина главного лепестка 


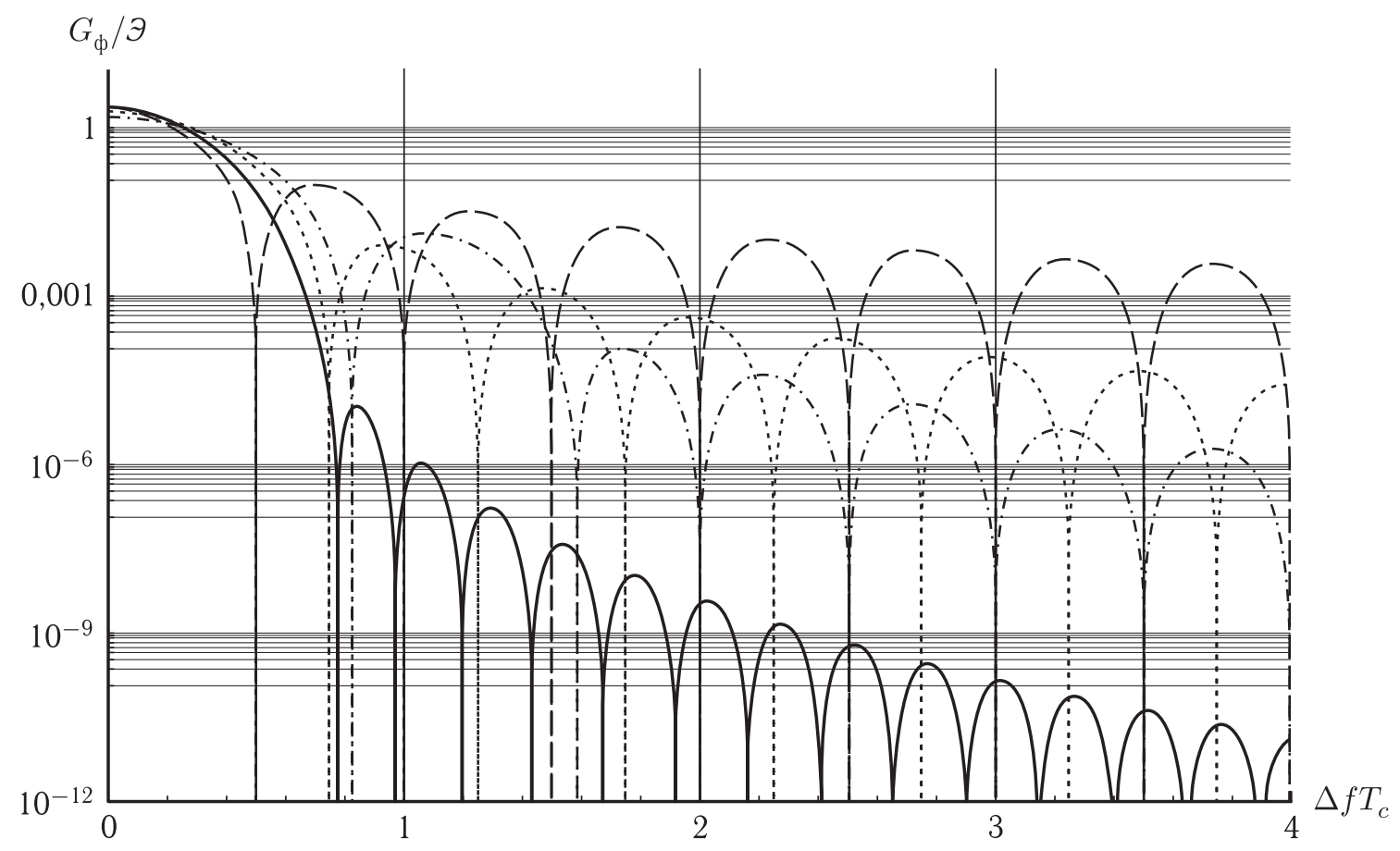

Рис. 4. Спектры сигналов: QBL-MSK (сплошная линия), гармонического ЧМ-MC (штрихпунктирная линия), классического и сформированного на основе частотной манипуляции с непрерывной фазой ЧМ-МС (точечная линия), ФМ-4 (пунктирная линия)

спектра мощности QBL-MSK-сигнала определяется значением $\Delta f T_{c}=0,77$, гармонического ЧМ$\mathrm{MC}$-сигнала значением $-\Delta f T_{c}=0,82$, классического ЧМ-MС-сигнала значением $-\Delta f T_{c}=$ $=0,75$ и ФМ-4-сигнала - значением $\Delta f T_{c}=$ $=0,5$. Максимум первого бокового лепестка энергетического спектра QBL-MSK-сигнала на 53 дБ меньше его главного максимума. Для классического ЧМ-МС-сигнала это соотношение составляет 23 дБ, для гармонического ЧМ-МС-сигнала $-20,6$ дБ, а для ФМ-4-сигнала - 13,5 дБ. Скорость убывания максимумов последующих лепестков спектра оказывается наибольшей у QBLMSK-сигнала и наименьшей у ФM-4-сигнала. Уже при расстройках относительно центральной частоты на величину $\Delta f T_{c} \geqslant 2$ максимумы лепестков спектра QBL-MSK-сигнала не менее чем на 40 дБ оказываются ниже максимумов лепестков спектра гармонического ЧМ-MC-сигнала, на 50 дБ ниже максимумов лепестков классического ЧМ-MCсигнала и на 64 дБ ниже максимумов лепестков ФМ-4-сигнала, что свидетельствует о высокой спектральной эффективности QBL-MSK-сигнала по сравнению с гармоническим ЧМ-MC, классическим ЧМ-MC и ФМ-4-сигналами.

\section{Выводы}

1. Представлена математическая модель незамирающего QBL-MSK-сигнала, на основе которой найден энергетический спектр этого сигнала и дано обоснование алгоритмов его формирования и приема.

2. Показано, что по спектральной эффективности QBL-MSK-сигнал превосходит классический ЧМ-МС-сигнал, ЧМ-МС-сигнал, сформированный на основе частотной манипуляции с непрерывной фазой, а также гармонический ЧМMC-сигнал и сигнал с четырехпозиционной фазовой манипуляцией, а по энергетической эффективности практически не отличается от них.

3. Полученные результаты могут оказаться полезными при создании систем цифровой радиосвязи, при обосновании рекомендаций по час- 
тотно-территориальному разносу радиоэлектронных средств различного назначения, а также при разработке различных вопросов радиоподавления средств радиосвязи.

\section{Список литературы}

1. Варгаузин Н.Т., Цикин И.А. Методы повышения энергетической и спектральной эффективности цифровой радиосвязи. СПб.: БХВ-Петербург, 2013.

2. Маковеева М.М., Шинаков Ю.С. Системы связи с подвижными объектами. М.: Радио и связь, 2002.

3. Aнтипенский Р.В., Ерзин И.Х., Поддубный В.Н. Спектральная эффективность и помехоустойчивость приема гармонического частотно-манипулированного сигнала с минимальным сдвигом // Радиотехника, 2012, № 5. С. 94-98.

4. Агафонов А.А., Каунов А.Е., Кондратенко А.Е., Ложкин К.Ю., Поддубный В.Н. Синтез некогерентного приемника простых частотно-манипулиро- ванных сигналов с минимальным сдвигом // Радиотехника, 2009, № 11. С. 40-57.

5. Агафонов А.А., Поддубный В.Н. Влияние группирования ошибок на помехоустойчивость приема частотно-манипулированного сигнала с минимальным сдвигом // Радиотехника, 1997, № 6. С. 47-50.

6. Грибанов В. В., Ерзин И.Х., Поддубный В.Н. Спектральная и энергетическая эффективность ЧМMC-сигнала, сформированного на основе частотной манипуляции с непрерывной фазой // Электромагнитные волны и электронные системы, 2015, № 1. C. $16-24$.

7. Гоноровский И.С. Радиотехнические цепи и сигналы. М.: Радио и связь, 1986.

8. Макаров С.Б., Цикин И.А. Передача дискретных сообщений по радиоканалам с ограниченной полосой пропускания. М.: Радио и связь. 1990.

9. Банкет В.Л., Дорофеев В.М. Цифровые методы в спутниковой связи. М.: Радио и связь, 1988.

10. Яглом А.М., Яглом И.М. Вероятность и информация. М.: Наука, 1975. 